\title{
Perfil do uso de medicamentos por idosos da Estratégia Saúde da Família de Porto Alegre
}

\section{Profile of drug use by the elderly of Family Health Program in Porto Alegre}

Como citar este artigo: ANDRADE, C. P.; ENCROFF, P.;

SCNAOLIN, V.; COMES, l.; TERRA, N. L. Perfil do uso de medicamentos por idosos da Estratégia Saúde da Família de Porto Alegre. Revista Saúde (Sta. Maria). 2019 45 (2).

\section{Autor correspondente:}

Nome: Vanessa Sgnaolin

E-mail: vanessa.sgnaolin@pucrs.br Telefone: (5 I) 3320.3000 (ramal 2660)

Formação Profissional: Formada(o) em Farmácia pela (o) Pontifícia Universidade Católica do Rio Grande do Sul (PUCRS), Porto Alegre, RS, Brasil. Doutora em Cerontologia Biomédica pela Pontifícia Universidade Católica do Rio Grande do Sul (PUCRS), Porto Alegre, RS, Brasil.

Filiação Institucional: Pontificia Universidade Católica do Rio Grande do Sul (PUCRS)

Endereço para correspondencia:

Rua: Av. Ipiranga n: 6681 -

Prédio 81 - $7^{\circ}$ andar, sala 703

Bairro: Jardim Botânico

Cidade: Porto Alegre Estado:

Rio Grande do Sul

CEP: 906 19-900

Data de Submissão:

21/05/2019

Data de aceite:

16/07/2019

Conflito de Interesse: Não há conflito de interesse

\section{RESUMO}

Objetivo: Avaliar o perfil farmacológico dos idosos atendidos pela Estratégia Saúde da Família (ESF). Métodos: Estudo transversal, coletado de forma prospectiva em uma amostra aleatória da população idosa cadastrada na ESF de Porto Alegre/RS. Os idosos foram entrevistados nas residências pelos agentes comunitários de saúde que aplicaram um questionário geral, contendo informações sobre medicamentos. Os dados farmacoterapêuticos foram utilizados para identificar os princípios ativos, classificados pela Anatomical Therapeutical Chemical Classification System. Resultados: Foram incluídos 761 participantes com idade média de $77,1 \pm 10,3$ anos, representados na sua maioria por mulheres $(63,9 \%)$, cor branca $(64,7 \%)$ e primeiro grau incompleto $(66,8 \%)$. A média de medicamentos utilizados foi de $4,1 \pm 3,1$ e a prevalência $85,0 \%$. Os grupos anatômicos mais utilizados foram: sistema cardiovascular $(80,1 \%)$, sistema digestivo e metabolismo $(56,9 \%)$ e sistema nervoso $(46,8 \%)$. Em relação ao entendimento, $75,4 \%$ dos idosos reconheciam o medicamento pelas características da embalagem e 53,4\% adquiriam na rede pública. Não aderiam ao tratamento farmacológico $66,8 \%$. Conclusão: Como os idosos são os que mais utilizam medicamentos, estudos com essa abordagem podem contribuir para formulação de estratégias de atenção a essa população.

PALAVRAS-CHAVE: Idoso; Uso de medicamentos; Terapêutica.

\section{ABSTRACT}

Objetive: To evaluate the pharmacological profile of the elderly attended by the Family Health Strategy (FHS). Methods: Cross-sectional study prospectively collected in a random sample of the elderly population registered in the FHS of Porto Alegre / RS. The elderly were interviewed by community health workers in homes who applied a general questionnaire containing information on drugs. The pharmacotherapeutic data were used to identify the active substances classified by the Anatomical Therapeutic Chemical Classification System. Results: Were included 761 participants with mean age of $77.1 \pm 10.3$ years, most of them by females $(63.9 \%)$, white $(64.7 \%)$ and incomplete primary school $(66.8 \%)$. The medication average was $4.1 \pm 3.1$ and the prevalence of use was $85.0 \%$. The most frequently used anatomical groups were: cardiovascular system (80.1\%), digestive system and metabolism (56.9\%) and nervous system (46.8\%). Regarding the prescription understanding, $75.4 \%$ recognized the drug by the characteristics of the packaging and $53.4 \%$ acquired in the public system. Pharmacological treatment was not adhered by $66.8 \%$. Conclusion: As the elderly are the most used drugs, studies with this approach can contribute to the formulation of strategies attention to this population.

KEYWORDS: Aged; Drug Utilization; Therapeutics. 


\section{INTRODUÇÃO}

O envelhecimento populacional vem crescendo no cenário mundial e assim a população hoje pode esperar viver além dos 60 anos. Combinado com quedas acentuadas nas taxas de fertilidade, esses aumentos na expectativa de vida levam ao rápido envelhecimento das populações em todo o mundo'. Segundo o Instituto Brasileiro de Geografia e Estatística (IBGE) a população com 60 anos ou mais de idade passou de 14,2 milhões, em 2000, para 19,6 milhões, em 2010, podendo ainda atingir 41,5 milhões em 2030, e ainda 73,5 milhões em $2060^{2}$.

O envelhecimento acarreta no surgimento de doenças crônico-degenerativas como diabetes mellitus, hipertensão arterial, demência entre outras, que necessitam de recursos farmacológicos em maior proporção e de variadas classes ${ }^{3}$. Devido às doenças crônicas outros fatores como idade, gênero e estado de saúde acabam fazendo com que os idosos pratiquem a polifarmácia, que possui uma alta prevalência nesta faixa etária 4 .

A Organização Mundial da Saúde (OMS) considera que mais de $50 \%$ dos medicamentos são prescritos ou dispensados de forma inadequada e que $50 \%$ dos pacientes tomam medicamentos de uma maneira incorreta ${ }^{5}$. Entre estas, a não adesão ao tratamento farmacológico implica em sérios agravos a saúde do idoso, pois interfere no tratamento de possíveis doenças presentes nestes indivíduos, consequentemente causando o agravo das mesmas. Outros fatores que também podem influenciar no uso inadequado dos medicamentos são: baixo nível socioeconômico, baixa escolaridade, relação com profissionais de saúde, doenças neurológicas em que podem afetar a cognição e a memória, restrições físicas e doenças crônicas ${ }^{6,7}$.

Estudos que avaliem o uso de medicamentos por indivíduos idosos são importantes para o desenvolvimento de estratégias de educação e prevenção de danos à sua saúde. Portanto, este estudo busca descrever o perfil farmacológico da população idosa atendida na Estratégia Saúde da Família (ESF) do município de Porto Alegre/RS.

\section{MÉTODO}

Desenho do Estudo

Estudo transversal, descritivo e analítico, onde foram coletados os dados de forma prospectiva em uma amostra aleatória da população idosa cadastrada na ESF do município de Porto Alegre/RS. Esta pesquisa fez parte do "Estudo Epidemiológico e Clínico dos Idosos atendidos pela Estratégia Saúde da Família do município de Porto Alegre - EMISUS”, realizado pelo Instituto de Geriatria e Gerontologia da Pontifícia Universidade Católica do Rio Grande do Sul (PUCRS), em parceria com a Secretaria Municipal de Saúde de Porto Alegre. 
Para a seleção desses idosos foram sorteadas 30 equipes da ESF, de forma estratificada por gerência distrital (GD). De cada ESF foram sorteados 36 idosos, correspondendo a um total de 1080 participantes.

\section{Coleta de Dados}

As coletas de dados foram realizadas no período de Março/2011 a Dezembro/2012, gerando um banco de dados com 761 participantes devido às perdas que ocorreram durante o desenvolvimento do estudo. Os idosos foram entrevistados em suas residências pelos agentes comunitários de saúde (ACS) que aplicaram um questionário geral, que incluiu dados demográficos e socioculturais, hábitos de vida, informações gerais sobre a saúde e uso de medicamentos.

\section{Critérios de inclusão}

Idade igual ou superior a 60 anos. Estar cadastrado em uma das ESF sorteadas para o estudo e concordarem em participar da pesquisa por meio da assinatura do Termo de Consentimento Livre e Esclarecido.

Variáveis em estudo

Os dados farmacoterapêuticos, obtidos do questionário geral foram utilizados para identificar os princípios ativos dos medicamentos usados pelos idosos participantes da pesquisa. Os princípios ativos presentes em cada especialidade farmacêutica foram listados e classificados de acordo com o Anatomical Therapeutical Chemical (ATC) Classification System 9 . Os medicamentos foram agrupados de acordo com o grupo principal que é representado por uma letra (A: Aparelho digestivo e metabolismo; B: Sangue e órgãos hematopoiéticos, C: Aparelho cardiovascular, D: Medicamentos dermatológicos, G: Aparelho geniturinário e hormônios sexuais, H: Preparações hormonais sistêmicas, excluindo hormônios sexuais e insulinas, J: Anti-infecciosos gerais para uso sistêmico, L: Agentes antineoplásicos e imunomoduladores, M: Sistema musculoesquelético, N: Sistema nervoso, P: Produtos antiparasitários, inseticidas e repelentes, Q: Uso veterinário, R: Aparelho respiratório, S: Órgãos dos sentidos, V: Vários) e corresponde ao grupo anatômico. Dos três grupos anatômicos mais utilizados foram classificados seus subgrupos terapêuticos (Grupo A: A02 - Antiácidos, medicamentos para tratamento da úlcera péptica e da flatulência; A03 - Agentes antiespasmódicos, anticolinérgicos e propulsivos; A06 - Laxativos; A07 - Antidiarreicos, agentes anti-inflamatórios e anti-infecciosos intestinais; A10a - Insulinas; A10b - hipoglicemiantes orais; A11 - Vitaminas; A12 - Suplementos minerais. Grupo C: C01 Terapêutica cardíaca; C02 -Anti-hipertensores; C03 - Diuréticos; C04 - Vasodilatadores periféricos; C05-Vasoprotetores; 
C07 - Betabloqueadores; C08 - Bloqueadores dos canais de cálcio; C09 - Agentes que atuam sobre o sistema reninaangiotensina; C10 - Hipolipemiantes. Grupo N: N02 - Analgésicos; N03 - Antiepiléticos; N04 -Antiparkinsônicos; N05a - Antipsicóticos; N05b - Ansiolíticos; N05c - Hipnóticos e sedativos; N06a - Antidepressivos; N07 - Outros do sistema nervoso).

\section{Análise Estatística}

O banco de dados foi digitado em duplicata no programa File Maker e estruturado no aplicativo Microsoft Exce/®. Aanálise dos dados foi realizada no Statistical Package for Social Sciences (SPSS) versão 17.0. Os dados foram descritos através de frequências, médias e desvios padrões.

\section{Considerações éticas}

Este estudo foi aprovado pelo Comitê de Ética em Pesquisa da Pontifícia Universidade Católica do Rio Grande do Sul e da Secretaria Municipal de Saúde de Porto Alegre, conforme parecer número 10/04967 e registro 499 do processo 001.021434.10.7, respectivamente. Todos os participantes foram informados de forma clara dos objetivos e dos métodos da pesquisa e assinaram o Termo de Compromisso Livre e Esclarecido, conforme as Diretrizes e Normas Regulamentadoras em Pesquisa, da Resolução n 466/12 do Conselho Nacional de Saúde do Ministério da Saúde.

\section{RESULTADO}

Os 761 indivíduos incluídos no estudo tinham idade entre 60 e 103 anos, com média de 77,1 \pm 10,3 anos, representados na sua maioria pelo sexo feminino $(63,9 \%)$ e de cor branca $(64,7 \%)$. A maioria destes idosos era casada (36,9\%), tinha o primeiro grau incompleto $(66,8 \%)$, apresentava nível salarial individual de até um salário mínimo $(57,6 \%)$ e familiar de um a três salários mínimos (52,2\%).

Em relação à análise farmacoterapêutica, a média de medicamentos utilizados foi de 4,1 $\pm 3,1$. A prevalência do uso de medicamentos foi de $85,0 \%$, totalizando 3120 medicamentos consumidos. O número máximo de medicamentos utilizado por um idoso foi 16 , sendo que $62,8 \%$ utilizavam de um a três medicamentos.

Os grupos anatômicos da ATCC mais utilizados foram: sistema cardiovascular (80,1\%), sistema digestivo e metabolismo $(56,9 \%)$ e sistema nervoso $(46,8 \%)$. Dentre esses, os subgrupos terapêuticos mais frequentes foram: 
antiácidos, medicamentos para tratamento da úlcera péptica e da flatulência $(29,0 \%)$, agentes que atuam sobre o sistema renina-angiotensina $(47,0 \%)$ e analgésicos $(21,7 \%)$ (Tabela 1). Os princípios ativos mais frequentemente utilizados foram: hidroclorotiazida (41,4\%), captopril $(39,9 \%)$, ácido acetilsalicílico (39,6\%), sinvastatina $(31,2 \%)$, omeprazol $(27,0 \%)$ e paracetamol $(24,0 \%)$ (Tabela 2).

Tabela 1. Frequência e percentagem de medicamentos utilizados pelos idosos das ESF.

\begin{tabular}{|c|c|c|}
\hline Classificação ATC & Frequência & $\%$ \\
\hline A - Sistema digestivo e metabolismo & 368 & 56,9 \\
\hline $\begin{array}{l}\text { A02 - Antiácidos, medicamentos para tratamento da úlcera } \\
\text { péptica e da flatulência }\end{array}$ & 221 & 29,0 \\
\hline A03 - Agentes antiespasmódicos, anticolinérgicos e propulsivos & 17 & 2,2 \\
\hline A06 - Laxativos & 6 & 0,8 \\
\hline $\begin{array}{l}\text { A07 - Antidiarreicos, agentes anti-inflamatórios e } \\
\text { anti-infecciosos intestinais }\end{array}$ & 13 & 1,7 \\
\hline A10a - Insulinas & 39 & 5,1 \\
\hline A10b - Hipoglicemiantes orais & 166 & 21,8 \\
\hline A11 - Vitaminas & 53 & 7,0 \\
\hline A12 - Suplementos minerais & 21 & 2,8 \\
\hline B - Sangue e órgãos hematopoiéticos & 271 & 41,9 \\
\hline C - Sistema cardiovascular & 518 & 80,1 \\
\hline C01 - Terapêutica cardíaca & 54 & 7,1 \\
\hline C02 - Anti-hipertensores & 19 & 2,5 \\
\hline C03 - Diuréticos & 325 & 42,7 \\
\hline C04 - Vasodilatadores periféricos & 1 & 0,1 \\
\hline C05 - Vasoprotetores & 1 & 0,1 \\
\hline C07 - Betabloqueadores & 189 & 24,8 \\
\hline C08 - Bloqueadores dos canais de cálcio & 108 & 14,2 \\
\hline C09 - Agentes que atuam sobre o sistema renina-angiotensina & 358 & 47,0 \\
\hline C10 - Hipolipemiantes & 210 & 27,6 \\
\hline D - Medicamentos dermatológicos & 9 & 1,4 \\
\hline G - Sistema geniturinário e hormônios sexuais & 10 & 1,5 \\
\hline $\begin{array}{l}\text { H - Preparações hormonais sistêmicas, excluindo } \\
\text { hormônios sexuais e insulinas }\end{array}$ & 33 & 5,1 \\
\hline $\mathrm{J}$ - Anti-infecciosos gerais para uso sistêmico & 17 & 2,6 \\
\hline L - Agentes antineoplásicos e imunomoduladores & 9 & 1,4 \\
\hline M - Sistema musculoesquelético & 128 & 19,8 \\
\hline N - Sistema nervoso & 303 & 46,8 \\
\hline N02 - Analgésicos & 165 & 21,7 \\
\hline N03 - Antiepiléticos & 31 & 4,1 \\
\hline N04 - Antiparkinsônicos & 6 & 0,8 \\
\hline
\end{tabular}




\begin{tabular}{lcc} 
N05a - Antipsicóticos & 21 & 2,8 \\
N05b - Ansiolíticos & 51 & 6,7 \\
N05c - Hipnóticos e sedativos & 3 & 0,4 \\
N06a - Antidepressivos & 115 & 15,1 \\
N07 - Outros do sistema nervoso & 12 & 1,6 \\
\hline P - Produtos antiparasitários, inseticidas e repelentes & 3 & 0,5 \\
\hline R - Sistema respiratório & 71 & 11,0 \\
\hline S - Sistema sensorial & 10 & 1,5 \\
\hline
\end{tabular}

As frequências foram calculadas com base no número total de pacientes ( $N=761)$.

Tabela 2. Frequência e percentagem de medicamentos utilizados pelos idosos das ESF

\begin{tabular}{lcc} 
Medicamentos & Frequência & $\%$ \\
\hline Hidroclorotiazida & 268 & 41,4 \\
\hline Captopril & 258 & 39,9 \\
\hline AAS & 256 & 39,6 \\
\hline Sinvastatina & 202 & 31,2 \\
\hline Omeprazol & 175 & 27,0 \\
\hline Paracetamol & 155 & 24,0 \\
\hline Metformina & 138 & 21,3 \\
\hline Propranolol & 98 & 15,1 \\
\hline Anlodipina & 87 & 13,4 \\
\hline Enalapril & 73 & 11,3 \\
\hline Glibenclamida & 73 & 11,3 \\
\hline Ibuprofeno & 72 & 11,1 \\
\hline Cálcio, carbonato de & 70 & 10,8 \\
\hline Furosemida & 52 & 8,0 \\
Fluoxetina & 51 & 7,9 \\
\hline
\end{tabular}

Em relação ao entendimento, $75,4 \%$ dos idosos reconheciam o medicamento pelas características da embalagem e 54,3\% entendiam o que está escrito em suas receitas (Tabela 3). Sobre o acesso aos medicamentos, $85,4 \%$ conseguiam seus medicamentos e 53,4\% adquiriam na rede pública (Tabela 3). Dos indivíduos analisados, 66,8\% não aderiam aos seus tratamentos farmacológicos (Tabela 4).

Tabela 3. Entendimento e acesso aos medicamentos utilizados pelos idosos das ESF.

\begin{tabular}{ccc} 
Entendimento & Frequência & $\%$ \\
\hline Como sabe que o medicamento é o certo? & & \\
\hline Características do comprimido & 110 & 18,1 \\
Características da embalagem & 459 & 75,4
\end{tabular}




\begin{tabular}{ccc} 
Alguém ajuda & 40 & 6,2 \\
\hline Entende o que está escrito na sua receita? & 268 & 45,7 \\
Não & 319 & 54,3 \\
Sim & Frequência & $\%$ \\
\hline Acesso & & \\
\hline Consegue todos os medicamentos que precisa tomar? & 90 & 14,6 \\
\hline Não & 528 & 85,4 \\
Sim & & \\
\hline De que forma consegue os seus medicamentos? & 90 & 14,5 \\
\hline Privado & 332 & 53,4 \\
Público & 200 & 32,2 \\
\hline Misto & & \\
\hline
\end{tabular}

Tabela 4. Adesão ao tratamento farmacológico.

\begin{tabular}{llc} 
Adesão medicamentosa & Frequência & $\%$ \\
\hline Morisky & & \\
\hline Adesão & 31 & 33,2 \\
Não adesão & 320 & 66,8 \\
\hline Alguma vez se esquece de tomar os seus medicamentos? & 52,3 \\
\hline Não & 292 & 47,7 \\
Sim & & \\
\hline É descuidado com os horários de tomar os seus medicamentos? & 422 & 70,8 \\
\hline Não & 174 & 29,2 \\
Sim & 489 & \\
\hline Para de tomar seus medicamentos quando está se sentindo melhor? & 121 & 19,8 \\
\hline Não & & \\
Sim & 470 & 78,2 \\
\hline Em algum momento se sentiu mal e parou de tomar seus medicamentos? & \\
\hline Não & 131 & 21,8 \\
\hline Sim & 40,2 \\
\hline
\end{tabular}

\section{DISCUSSÃO}

O presente estudo descreveu o perfil de utilização de medicamentos pelos idosos das equipes da ESF de Porto Alegre. Os resultados demonstraram uma alta prevalência de idosos que utilizavam medicamentos para o sistema cardiovascular, indicando que estes possuíam alguma doença relacionada. Os medicamentos mais utilizados pelos indivíduos analisados são indicados para o tratamento de hipertensão arterial sistêmica (HAS), doença que mais acomete os idosos, como citado por Pimenta et. al. ${ }^{10}$ que demonstraram uma frequência de $72 \%$ de idosos com HAS assim como 
em outros estudos ${ }^{11,12}$. Estes dados levam a uma grande preocupação, pois a HAS não controlada pode gerar alguns fatores de risco como complicações cardíacas, cerebrovasculares e renais ${ }^{13}$. Outro fator importante a ser considerado sobre os medicamentos cardiovasculares, é que estes são responsáveis pelas maiores frequências de interações medicamentosas e efeitos adversos, bem como necessitam de uma criteriosa avaliação do seu risco/benefício ${ }^{14,16}$.

Outro dado relevante foi a alta prevalência dos medicamentos para o sistema digestivo e metabolismo como os hipoglicemiantes orais, que são indicados para tratamento da diabetes mellitus, os antiácidos e os antiulcerosos. Estudos como o de Vitoi et. al. ${ }^{17}$, encontraram uma prevalência de $22,4 \%$ dos idosos com diabetes mellitus, demonstrando uma alta prevalência de diabéticos nesta faixa etária, o que vem ao encontro da grande utilização de hipoglicemiantes orais. Os antiácidos e antiulcerosos são muito utilizados através da automedicação, sendo este fato preocupante, pois podem causar interações medicamentosas com outros fármacos como o captopril, segundo medicamentos mais citado neste estudo. A associação de captopril com antiácidos pode causar a redução do efeito hipotensor ${ }^{18}$. Scherer et al. ${ }^{3}$ também demonstraram uma alta prevalência do uso de antiácidos e antiulcerosos, ainda que menor do que encontrado em nosso estudo. Já Santos et al. ${ }^{19}$ encontraram um baixo consumos desta classe, apenas $4,1 \%$. Os antiulcerosos podem causar, além de alterações na absorção de outros fármacos, depleção de fósforo e de cálcio, o que é mais preocupante nestes indivíduos, pois é muito comum nesta faixa etária possuírem rápida degeneração óssea ${ }^{20,21}$. 0 omeprazol é um dos antiulcerosos mais utilizados e o mais citado pelos indivíduos deste estudo. O seu uso prolongado pode estar associado com o número de medicamentos prescritos ${ }^{22}$.

Devido às doenças que surgem durante o envelhecimento os idosos podem relatar a presença de dores, que muitas vezes se tornam crônicas, e este fato pode levar ao uso de analgésicos ${ }^{23}$. Nosso estudo demonstrou uma elevada utilização de medicamentos para o sistema nervoso, sendo os analgésicos os mais utilizados. Oliveira et. al. ${ }^{24}$ também encontrou dados semelhantes e ressaltou a automedicação como a causa desta grande utilização de analgésicos por idosos.

Os antidepressivos foram uma das classes medicamentosas mais utilizadas, sendo a fluoxetina o mais citado pelos idosos. Este dado revela um importante problema de saúde pública, sendo que este medicamento é considerado potencialmente inapropriado de acordo com a lista PRISCUS. O principal fator é que este medicamento pode causar efeitos secundários do sistema nervoso central (náuseas, insônia, tonturas, confusão) e hiponatremia nos idosos. A lista PRISCUS apresenta como alternativas terapêuticas mais seguras para o tratamento da depressão como outros inibidores seletivos de recaptação de serotonina (sertralina, citalopram), trazodona, mirtazapina ou tratamentos não farmacológicos tais como terapia comportamental ${ }^{25}$.

Os medicamentos mais frequentes citados pelos idosos estão disponíveis pelo Sistema Único de Saúde (SUS), também presentes na Relação Municipal de Medicamentos (REMUME) de Porto Alegre. Este fato reafirma que a rede 
pública foi a forma de aquisição dos medicamentos mais utilizada pelos idosos. Isto também justifica a prescrição de fluoxetina para os idosos, mesmo sendo considerada inapropriada para este grupo etário, pois está disponível pelo SUS na REMUME. É importante destacar que apesar da fluoxetina não ser indicada para esta faixa etária, deve-se considerar que é preferível o seu uso ao invés do não tratamento, já que trata-se de uma população de baixa renda.

Ao avaliar as situações que envolvem o entendimento do uso dos medicamentos observou-se que a maioria reconhece estes através das características da embalagem. No Art. 17 da RDC № 71/2009 fica estabelecido que não podem constar nos rótulos dos medicamentos informações tais como símbolos, figuras e representações gráficas, que possam gerar má interpretação ou equívoco na utilização dos mesmos ${ }^{26}$. Outra consideração em relação à população estudada é que os idosos apresentavam baixa escolaridade, o que pode impactar na dificuldade de identificação do medicamento por meio da leitura, tanto das informações contidas na embalagem quanto da receita médica. Isso representa um risco à saúde, pois pode ocorrer troca de medicamento e seu uso incorreto. Condições clínicas relacionadas ao envelhecimento tais como a diminuição da visão, presença de quadros demenciais e depressivos que levam à diminuição da cognição e consequentemente esquecimento, podem estar associados a erros na administração dos medicamentos ${ }^{27}$.

Em relação a adesão ao tratamento farmacológico denota-se um percentual elevado de não aderência. Este resultado coincide com os dados publicados no estudo de Ramos et. al. ${ }^{28}$, realizado com 356 indivíduos com 60 anos ou mais idades, no município de Caxias - Maranhão, que encontraram o percentual de $67,3 \%$ de não aderência, assim como Rocha et al. ${ }^{29}$, que relataram uma prevalência de $62,9 \%$ dos idosos pesquisados como sendo não aderentes ao tratamento. O principal motivo encontrado no presente estudo foi o esquecimento, que pode ter sido potencializado pelas características da população, como baixa escolaridade e presença de doenças neuropsiológicas ${ }^{30}$. Esta não adesão pode prejudicar o tratamento proposto, o que enfatiza a importância de profissionais da saúde definirem uma estratégia para prescrição e para a orientação ao paciente.

\section{CONSIDERAÇÕES FINAIS}

Os idosos são a população que mais utiliza medicamentos, por isso estudos com esse enfoque podem contribuir com estratégias de atenção, educação e prevenção a essa população, principalmente na ESF. Dados epidemiológicos e farmacológicos são fundamentais para o desenvolvimento de um plano de atuação, onde a inclusão de farmacêuticos nessas equipes é de uma imensa importância. Estes profissionais auxiliam no cuidado ao paciente, prestando a assistência farmacêutica, e fornecem orientações farmacológicas, como o modo de uso e a importância da utilização correta dos medicamentos, buscando melhorar a adesão terapêutica.

Percebe-se também a importância de novas atualizações na lista REMUME, visando incluir medicamentos mais 
adequados aos idosos, que possuem algumas limitações fisiológicas que podem interferir na resposta a determinados medicamentos. Assim, uma escolha farmacológica adequada torna a terapia eficaz e gera uma melhor qualidade de vida para o idoso.

\section{REFERÊNCIAS}

1. Organização Mundial da Saúde. Relatório mundial de Envelhecimento e Saúde, 2015. [acessado 2019 mai. 21]. Disponível em: <https://sbgg.org.br/wp-content/uploads/2015/10/OMS-ENVELHECIMENTO-2015-port.pdf>.

2. Instituto Brasileiro de Geografia e Estatística. Estudos e Análises. Mudança demográfica no Brasil no início do século XXI. Subsídios para projeções da população. Rio de Janeiro 2015. [acesso 2019 mai. 19]. Disponível em: <http://biblioteca.ibge.gov.br/visualizacao/livros/liv93322.pdf>.

3. Scherer VC, Weber BR, Oliveira KR. Perfil dos medicamentos utilizados por idosos atendidos em uma farmácia comunitária do município de ljuí/RS. Rev. Contexto \& Saúde. 2011;10(20):375-84.

4. Hovstadius B, Petersson G. Factorsleanding to excessive polypharmacy. Clin Geriatr Med. 2012;28(2):15972.

5. World Health Organization. The safety of medicines in public health programmes: pharmacovigilance an essential tool. Geneva2006. [acessado 2019 mai 20]. Disponível em: <http://www.who.int/medicines/areas/quality_safety/ safety_efficacy/Pharmacovigilance_B.pdf>.

6. Schlenk EA, Dunbar JJ, Engberg S. Medication non-adhrence among older adults: a review of strategies and interventions for improvement. J Gerontol Nurs. 2004;30(7):33-43.

7. Rozenfeld S. Prevalência, fatores associados e mau uso de medicamentos entre os idosos: uma revisão. Rev Saude Publica. 2003;19(3):717-24.

8. Gomes I, Nogueira EL, Engroff P, Ely LS, Schwanke CHA, De Carli GA, et al. The multidimensional study 
of the elderly in the family health strategy in Porto Alegre, Brazil (EMI-SUS). PAJAR. 2013;1(1):20-4.

9. WHO. The Anatomical Therapeutic Chemical Classification System 2019. [acesso 2019 mai 10]. Disponível em: http://www.whocc.no/atc_ddd_index/.

10. Pimenta FB, Pinho L, Silveira MF, Botelho ACC. Fatores associados a doenças crônicas em idosos atendidos pela estratégia de saúde da família. Cien Saude Colet. 2015;20(8):2489-98.

11. Marin MJS, Cecílio LCO, Perez AEWUF, Santella F, Silva CBA, Filho JRG, et al. Caracterização do uso de medicamentos entre idosos de uma unidade do Programa Saúde da Família. Cad Saude Publica. 2008;24(7):1545-55.

12. Bezerra TA, Brito MAA, Costa KNFM. Caracterização do Uso de Medicamentos Entre Idosos Atendidos em Uma Unidade Básica de Saúde da Família. Cogitare Enferm. 2016;21(1):1-11.

13. Menezes TN, Oliveira ECT, Fischer MATS, Esteves GH. Prevalência e controle da hipertensão arterial em idosos: um estudo populacional. Rev Port Saúde Pública. 2016;34(2):117-24.

14. Mosegui GBG, Rozenfeld S, Veras RP, Vianna CMM. Avaliação da qualidade do uso de medicamentos em idosos. Rev Saude Publica. 1999;33:437-44.

15. Miranda RD, Perrotti TC, Bellinazzi VR, Nobrega TM, Cnederoglo MS, Toniolo Neto J. Hipertensão arterial no idoso: peculiaridades na fisiopatologia, no diagnóstico e no tratamento. Rev Bras Hipertens. 2002;9:293-300.

16. Morsch LM, Dressler CC, Schneider APH, Machado EO, Assis MP. Complexidade da farmacoterapia em idosos atendidos em uma farmácia básica no sul do Brasil. Infarma. 2015;27(4):239-47.

17. Vitoi NC, Foga AS, Nascimento CM, Franceschini SCC, Ribeiro AQ. Prevalência e fatores associados ao diabetes em idosos no município de Viçosa, Minas Gerais. Rev Bras Epidemiol. 2015;18(4):953-65.

18. Egger SS, Drewe J, Schlienger RG. Potential drug-drug interactions in the medication of medical patients at hospital discharge. Eur J Clin Pharmacol. 2003;58(11):773-8. 
19. Santos TRA, Lima DM, Nakatani AYK, Pereira LV, Leal GS, Amaral RG. Consumo de medicamentos por idosos, Goiânia, Brasil. Rev Saude Publica. 2013;47(1):94-103.

20. Fonseca AL. Interações medicamentosas. 3. edição. Rio de Janeiro: EPUB; 2000.

21. Merck SD. Manual Merck de informação médica. São Paulo: Manole; 2002.

22. Hipólito P, Rocha BS, Oliveira FJAQ. Perfil de usuários com prescrição de omeprazol em uma Unidade Básica de Saúde do Sul do Brasil: considerações sobre seu uso racional. Rev Bras Med Fam Comunidade. 2016;11(38):110.

23. Jones MR, Ehrhardt KP, Ripoll JG, Sharma B, Padnos IW, Kaye RJ, et al. Pain in the Elderly. Curr Pain Headache Rep. 2016;20(4):23.

24. Oliveira MA, Francisco PMSB, Costa KS, Barros MBA. Automedicação em idosos residentes em Campinas, São Paulo, Brasil: prevalência e fatores associados. Cad Saude Publica. 2012;28(2):335-45.

25. Holt S, Schmiedl S, Thürmann PA. Potentially inappropriate medications in the elderly: the PRISCUS List. Dtsch Arztebl Int. 2010;107(31-32):543-51.

26. BRASIL. ANVISA. Agência Nacional de Vigilância Sanitária. Resolução RDC nº 71, de 22 de dezembro de 2009. Estabelece regras para a rotulagem de medicamentos. [acesso 2019 mai 01]. Disponível em: <http://bvsms. saude.gov.br/bvs/saudelegis/anvisa/2009/res0071_22_12_2009.html>.

27. Bertoldi AD, Barros AJD, Hallal PC, Lima RC. Utilização de medicamentos em adultos: prevalência e determinantes individuais. Rev Saude Publica. 2004;38(2):228-38.

28. Ramos JS, Carvalho Filha FSS, Silva RNA. Avaliaçãoda adesão ao tratamento por idosos cadastrados no programa do hiperdia. Rev Gestão Sistemas Saúde. 2015;4(1):29-39.

29. Rocha CH, Oliveira APS, Ferreira C, Faggiani FT, Schroeter G, Souza ACA, et al. Adesão à prescrição 
médica em idosos de Porto Alegre, RS. Cien Saude Colet. 2008;13(Sup):703-710.

30. Nogueira EL, Rubin LL, Giacobbo SS, Gomes I, Cataldo Neto A. Screening for depressive symptoms in older adults in the Family Health Strategy, Porto Alegre, Brazil. Rev Saude Publica. 2014;48(3):368-77. 